\title{
Gyrochronology and its usage for main sequence field star ages
}

\author{
Sydney A. Barnes \\ Lowell Observatory, \\ 1400 W. Mars Hill Road, Flagstaff, AZ 86001, USA \\ email: barnes@lowell.edu
}

\begin{abstract}
The construction of all age indicators consists of certain basic steps which lead to the identification of the properties desirable for stellar age indicators. Prior age indicators for main sequence field stars possess only some of these properties. The measured rotation periods of cool stars are particularly useful in this respect because they have well-defined dependencies that allow stellar ages to be determined with $\sim 20 \%$ errors. This method, called gyrochronology, is explained informally in this talk, shown to have the desired properties, compared to prior methods, and used to derive ages for samples of main sequence field stars.
\end{abstract}

Keywords. stars: activity, stars: binaries, stars: evolution, stars: fundamental parameters, stars: individual ( $\xi$ Boo, $61 \mathrm{Cyg}, \alpha \mathrm{Cen}, 36 \mathrm{Oph})$, stars: late-type, stars: rotation

\section{Motivations}

Things in our world come into being, exist in time, and eventually cease to be. The properties of these things usually change over time, so that specifying the age of something, whether it be a tree, a human being, or a star, immediately gives us a good idea of what some of its other properties might be. In galactic astronomy, the ages of individual stars assume a particular importance, because they constitute the ticks of the abstract cosmic clock that tells us how various astronomical phenomena change over time.

Sandage (1962, and earlier) first noted that the morphology of a cluster of stars in the Hertzsprung-Russell diagram might be used to derive its age. Demarque \& Larson (1964) improved it substantially, and named it the isochrone method. Although venerable, this method is not very effective for main sequence stars because its principal variable, a star's luminosity, changes only slowly on the main sequence. Furthermore, for a field star, it also requires an excellent distance measurement, not easily accomplished. Consequently, isochrone ages for main sequence field stars have errors approaching $~ 100 \%$.

Of the prior distance-independent methods, the most consistent relies on the declining chromospheric activity of a cool star (Wilson, 1963; Skumanich 1972; Noyes et al. 1984; Soderblom et al. 1991; Donahue 1998). However, chromospheric emission varies with a star's rotation phase, activity cycle phase, and possibly other variables, limiting the precision of such ages to $\sim 50 \%$.

All activity-related age indicators are ultimately related to the rotation rate of a star. However, attempts to harness rotation to derive ages were hindered by the ambiguity of $v \sin i$ measurements, and what we now know to be a dynamo-related bimodality, and associated transition, in very young stars. The $v \sin i$ ambiguity can be entirely circumvented by (precisely) measuring a star's (mass-dependent) rotation period instead, and the early bimodality can be identified, and related stars excised.

This method, named gyrochronology (and parsed gyros-chronos-logos) allows the derivation of a significantly more precise age than previously available, of a cool main sequence 
field star, from its measured color and rotation period. The period is typically determined from time-series measurements of the spot-related photometric modulation of starlight. This talk is an informal summary of this method, as detailed in Barnes (2007). Some results and terminology derive from the 'CgI scenario' for stellar rotation presented in Barnes (2003).

\section{Background for the construction of all age indicators}

Many of the issues in constructing age indicators are so obvious that they are routinely ignored! Let us therefore proceed by first stepping back, and considering the main steps in the construction of any age indicator. One needs to:

(a) Find an observable, $v$, that changes 'well' with age; ('Well' means that it works for single objects rather than for an ensemble, and also has the properties listed in Table 1.)

(b) Determine the ages of suitable calibrators independently; (This means measuring both the variable, $v$, for the calibrating objects, and the most trustworthy prior variables so that $v$ can be related to earth rotations, pendulum swings, etc.)

(c) Measure the functional form of the variable: $v=v(t, w, x, \ldots)$; $(t$ represents the age, and $w, x, \ldots$ additional dependencies. Variables with the fewest dependencies are the most desirable.)

(d) Invert that functional form to find $t=t(v, w, x, \ldots)$; (Analytic inversions provide insight, but numerical inversions are usually necessary.)

(e) Calculate the error: $\delta t=\delta t(t, v, w, x, \ldots$.$) ; (Although necessary, non-linearities$ and other complexities often make this final step difficult.)

Table 1. Characteristics of the three major age indicators for field stars

\begin{tabular}{|c|c|c|c|}
\hline Property $\Downarrow$ & Isochrone Age & Chromospheric Age & Gyrochronology \\
\hline Measurable easily? & ? (Distance reqd.) & ? (Repetition reqd.) & ? (Repetition reqd.) \\
\hline Sensitive to age? & No (on MS) & Yes & Yes \\
\hline Insensitive to other parameters? & No & Yes & Yes \\
\hline Technique calibrable? & Yes (Sun) & $?($ Sun?) & Yes (Sun) \\
\hline Invertible easily? & No & Yes & Yes \\
\hline Errors calculable/provided? & $?$ (Difficult) & Yes? & Yes \\
\hline Coeval stars yield the same age? & No (Field binaries) & $?$ & Yes \\
\hline
\end{tabular}

The foregoing, and other practical considerations, suggest that the following properties are desirable for stellar age indicators.

(a) Measurability for single stars: The indicator should be properly defined, measurable easily itself, and preferably should not require many additional quantities to be measured, otherwise it cannot be used routinely.

(b) Sensitivity to age: The indicator should change substantially (and preferably regularly) with age, otherwise the errors will be inherently large.

(c) Insensitivity to other parameters: The indicator should have insensitive (or separable) dependencies on other parameters that affect the measured quantity, otherwise there is the potential for ambiguity. In particular, distance-independent methods are preferred.

(d) Calibration: The technique should be calibrable using an object (or set of objects) whose age(s) we know very well, otherwise systematic errors will be introduced. 
(e) Invertibility: The functional dependence determined above should be properly invertible to yield the age as a function of the measured variables.

(f) Error analysis: The errors on the age derived using the technique ought to be calculable, otherwise no confidence can be attached to the ages.

(g) Test of coeval stars: The technique should yield the same ages for stars expected to be coeval, otherwise the validity of the technique itself must be questioned.

Table 1 summarizes the extent to which these properties are satisfied for the three major field star age indicators now available.

\section{Introduction to rotational ages}

Skumanich (1972) seems to have been the first to identify a relationship between the rotation rate of a star and its age. He used the averaged $v \sin i$ values of stars in selected open clusters, and that of the Sun, all of whose ages are known independently. It was not clear then that such a relationship could be used in any more than a statistical sense, partly due to the inherent ambiguity in $v \sin i$ measurements. Observations of $v \sin i$ values, and later, rotation periods in young open clusters revealed a wide dispersion in the rotation rates of coeval stars that discouraged the use of rotation as an age indicator.

A prescient attempt was made by Kawaler (1989) to use rotation to derive ages based on the Hyades rotation period sequence, but its reliance on various theoretically motivated assumptions, the poor fit to the warm Hyades stars and the rotational dispersion in young open clusters cast doubts on its viability.

However, the availability of large numbers of rotation periods in open clusters allowed the resolution of this 'dispersion' into distinct rotational sequences, C \& I, in color-period diagrams, each with its own set of dependencies (Barnes 2003). This resolution shows that the (largely slower-rotating) I sequence does indeed spin down similar to Skumanich's initial suggestion, but the (largely faster-rotating) C sequence does not. However, C sequence stars change into I sequence stars within a couple of $100 \mathrm{Myr}$, so that all older cool stars must be of the I type, and spin down predictably. Furthermore, the spindown is convergent, in the sense that initial variations become increasingly unimportant with the passage of time.

These facts allow one to identify the principal dependencies of stellar rotation, which turn out to be stellar color/mass and age, and to identify empirically the tight relationship between them. This relationship must be true for all cool stars on the main sequence. Therefore, measuring a field star's color/mass and rotation period at once allow the age to be determined.

Furthermore, this method of determining the age is such that most of the properties considered desirable for an age indicator, as listed above, can be shown to be satisfied. Therefore, it seems appropriate to name the method 'gyrochronology.'

\section{Color-period diagrams}

Gyrochronology is ultimately based on color-period diagrams of open clusters, such as those shown in Fig. 1. (More such diagrams can be found in the papers by Meibom and Irwin in these proceedings.) The older ( 600 Myr-old) Hyades cluster shows a distinct diagonal sequence, called I, of faster-rotating warmer stars and slower-rotating cooler stars, marked with circles. The way to understand the color-period diagram of the younger ( $\sim 300$ Myr-old) cluster NGC 3532 is to realize that not only is this I sequence also present in this cluster, but another sequence, $C$, of faster-rotating stars, marked with asterisks. 


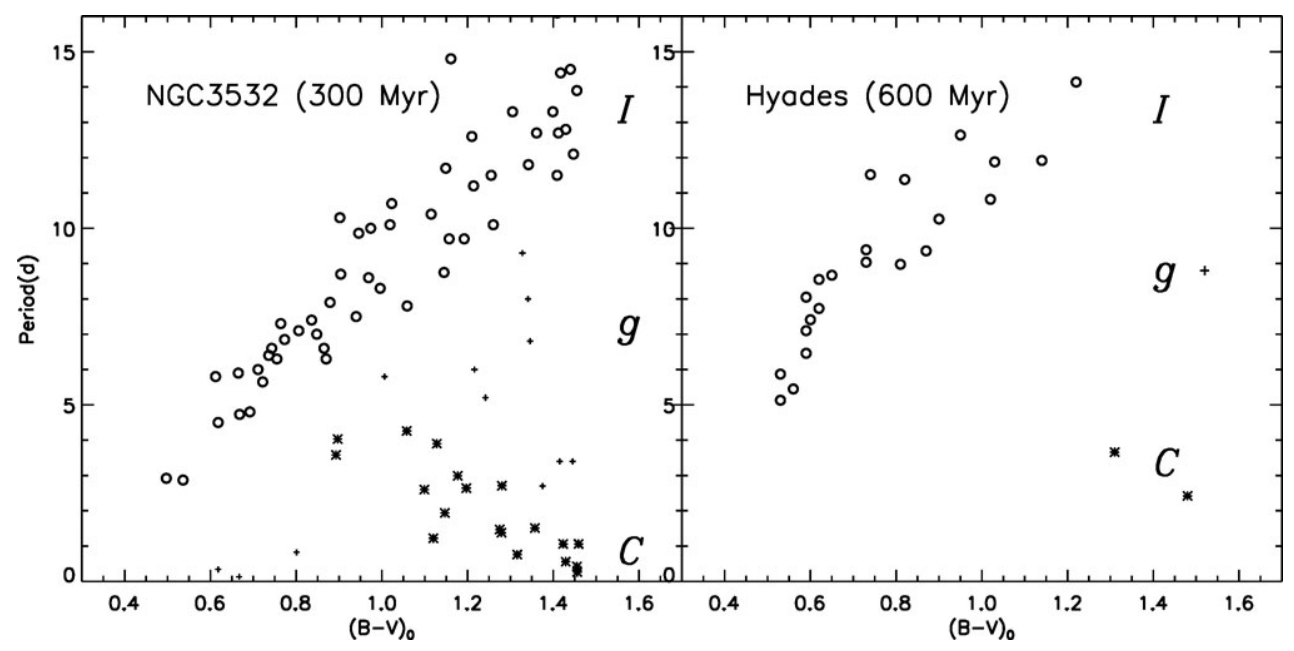

Figure 1. Color-period diagrams of the 300 Myr-old NGC3532 (Barnes, 1998) and the 600 Myr-old Hyades open clusters (primarily Radick et al. 1987). We see that the C-type stars in NGC 3532 (asterisks) have changed into I-type stars (circles) by Hyades age.

[The sequences are striking in the richer M 35 cluster (Meibom et al. 2008).] Comparing the two color-period diagrams tells us that almost all the C-type stars change into I-type stars by Hyades age. The stars in the rotational gap, g, between the two sequences can now be interpreted as stars in transition from the C- to the I-sequence.

These color-period diagrams also suggest that the color/mass dependence of the I sequence is the same for both clusters. This implies that the rotation period, $P$, of a star on this I sequence is expressible as the separable product of this mass dependence and of other variables, of which we might guess that the most important is the age, $t$, because stars spin down over time. Thus, we write $P=f(B-V) \cdot g(t)$.

\section{The dependencies of I sequence stars}

What might the age dependence, $g(t)$, be? A very good guess would simply be $g(t)=$ $\sqrt{t}$, in agreement with the original suggestion by Skumanich (1972). Indeed, when the rotation periods, $P$, of stars in all measured open clusters are divided by $g(t)=\sqrt{t}$, the I sequences are brought into coincidence, as shown in Fig. 2, from Barnes (2007). (These early data include binaries, possibly aliased periods and other pathologies, hence the scatter.)

Fig. 3 shows a similar coincidence for field stars, the single unevolved set of Mt. Wilson stars from Baliunas et al. (1996). We have used individual chromospheric ages calculated using the prescription of Donahue (1998). It is obvious that the C sequence stars in the younger open clusters have all changed into I sequence stars in the older Mt. Wilson sample. Furthermore, by guessing the age dependence, $g(t)$, using Skumanich (1972), the mass dependence of the I sequence has been made manifest.

Indeed, one can fit this dependence using a function of the form

$$
f(B-V)=a(B-V-c)^{b} \quad \text { giving } \quad a=0.773 \pm 0.011, b=0.601 \pm 0.024 .
$$

The translational term, $c$, was simply equated to 0.4 in Barnes (2007) and to 0.5 in Barnes (2003). A subsequent fit by Meibom et al. (2008), using both a large sample of 


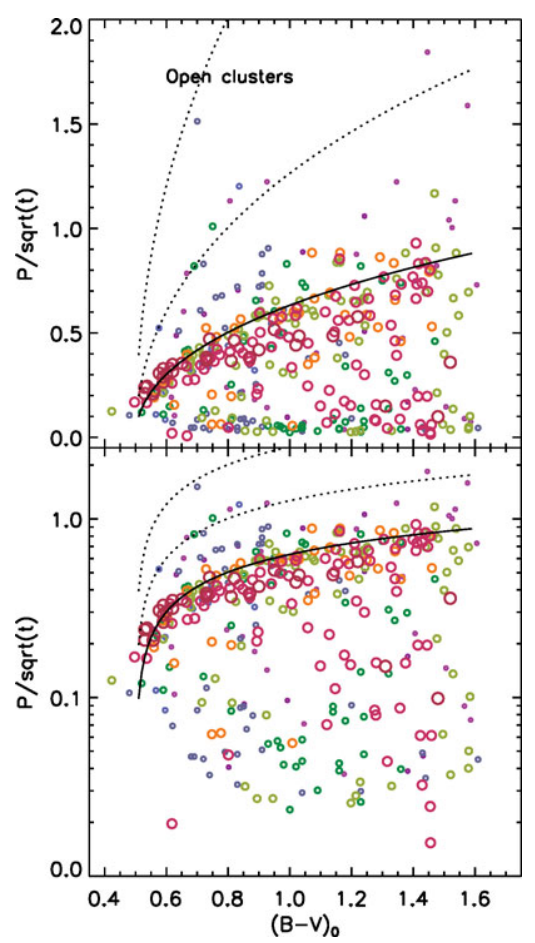

Figure 2. Rotation periods of cluster stars divided by the square roots of the cluster ages. Note the presence of both I sequence stars near the solid line, and $\mathrm{C}$ sequence stars below. (Figure from Barnes, 2007)

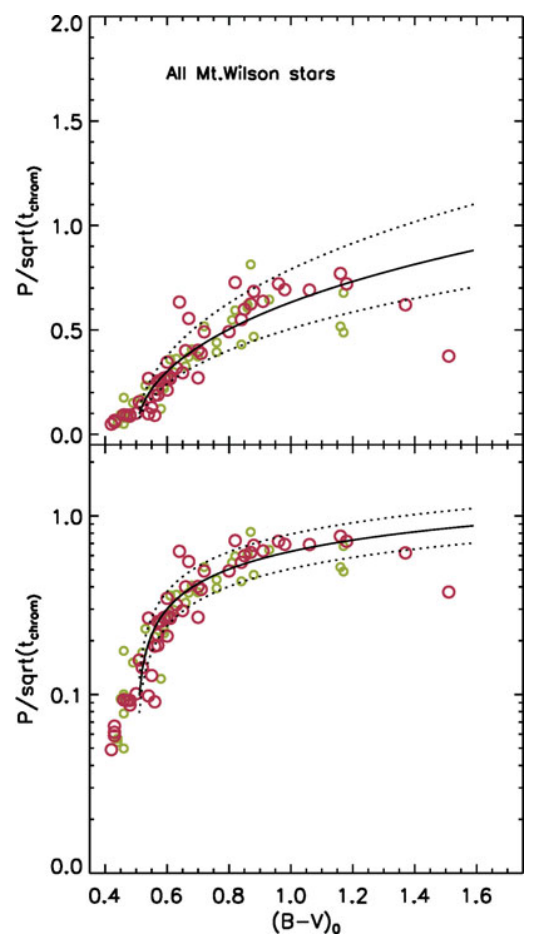

Figure 3. Rotation periods of single, main sequence Mt. Wilson stars, divided by the square roots of their chromospheric ages. Note that only I sequence stars are present. (Figure from Barnes, 2007)

rotation periods in the open cluster M35, and spectroscopic membership information, gives

$$
a=0.770 \pm 0.014, b=0.553 \pm 0.052, c=0.472 \pm 0.027 .
$$

The point is that regardless of the exact functional form chosen, a 2-3 parameter fit will suffice, and those parameters will be determined with small errors.

One final step of the construction remains. Having specified $f(B-V)$, we now return to $g(t)$. It is reasonable to seek a power law dependence: $g(t)=t^{n}$. This allows us to calibrate the method using the Sun by ensuring that the above mass dependence gives the Solar rotation period at Solar age. This calibration gives $n=0.519 \pm 0.007$.

Thus, the age of a star (in Myr) is simply given by inverting $P=f(B-V) . g(t)$ to get

$$
\log \left(t_{\text {gyro }}\right)=\frac{1}{n}\{\log P-\log a-b \times \log (B-V-c)\}
$$

where the constants $a, b, c, n$ are as specified above, and base 10 logarithms are used.

\section{Age error analysis}

A virtue of the above formulation is that the age error can be simply calculated, and the various contributing error terms seen in perspective. The expression for the fractional 
age error, as calculated in Barnes (2007), is:

$$
\frac{\delta t}{t}=2 \% \times \sqrt{3+\frac{1}{2}(\ln t)^{2}+2 P^{0.6}+\left(\frac{0.6}{x}\right)^{2}+(2.4 \ln x)^{2}}
$$

where $x=B-V-0.4$. For 1 Gyr-old stars of spectral types late F, early $\mathrm{G}$, mid $\mathrm{K}$ and early $\mathrm{M}$ respectively, we get

$$
\frac{\delta t}{t}=2 \% \times \begin{cases}\sqrt{26.9+6.4+66.5} & \text { when } B-V=0.5(P=7 d) \\ \sqrt{26.9+8.9+16.9} & \text { when } B-V=0.65(P=12 d) \\ \sqrt{26.9+12.1+2.5} & \text { when } B-V=1.0(P=20 d) \\ \sqrt{26.9+15.4+0.35} & \text { when } B-V=1.5(P=30 d)\end{cases}
$$

which shows the relative contributions of the period and color errors (second and third terms, respectively). Color errors and differential rotation are the significant contributors for bluer and redder stars respectively.

The expression above evaluates to fractional age errors of $13-20 \%$ for 1 Gyr-old early Mlate F stars, suggesting that relatively precise ages may indeed be derived for field stars, provided that the observable inputs, color and rotation period, are measured well.

\section{Application to field star samples}

Expressions (5.3) above and (6.1), for the gyro age and its error, respectively, are true for all I-type main sequence late F-early M stars, whether in clusters or in the field. We can therefore apply them to field star samples with measured rotation periods to derive ages where none were available before. The field star sample of Strassmeier et al. (2000) is an example.

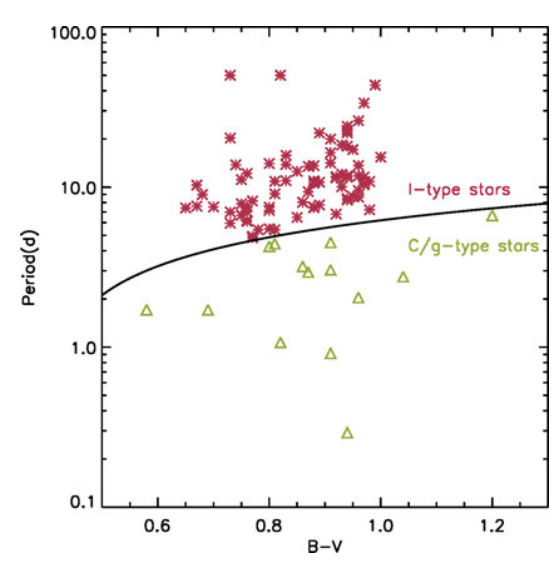

Figure 4. Color-period diagram for the Strassmeier et al. (2000) stars, showing the $100 \mathrm{Myr}-\mathrm{old}$ (gyro) isochrone used to discard possible C/g-type stars. Only the I-type stars above are retained for gyrochronology. (Figure from Barnes, 2007)

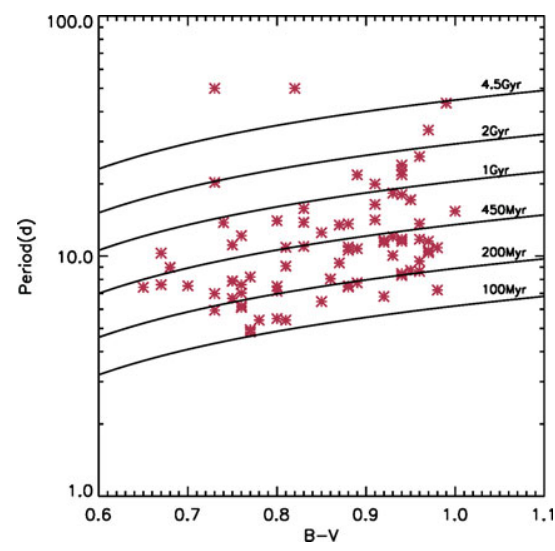

Figure 5. Rotational isochrones are shown for ages ranging from $100 \mathrm{Myr}$ to 4.5 Gyr for the I-type stars in the Strassmeier et al. (2000) sample. Note the relative youth of the sample, in keeping with its active pedigree. (Figure from Barnes, 2007)

Fig. 4 displays the color-period diagram for this sample, and the $100 \mathrm{Myr}$ (gyro) isochrone used to choose only the I-type stars for age analysis. Fig. 5 displays isochrones spanning the age range of the sample, showing its relative youth. Indeed, the median age 
is only $365 \mathrm{Myr}$, in agreement with the selection of the original sample by activity. The stars are individually tabulated in Barnes (2007), where the technique is also applied to the older sample of stars (median age of $1.2 \mathrm{Gyr}$ ) assembled by Pizzolato et al. (2003). For both samples, activity indicators like $R_{H K}$ and $L_{X} / L_{b o l}$ are found to decline as expected with increasing gyro age.

\section{Comparison with chromospheric ages}

The best age indicator for nearby field stars over the past couple of decades has been the decline of chromospheric emission with age. The calibrations regularly used are those of Soderblom et al. (1991) and Donahue (1998), but see Mamajek \& Hillenbrand (2008) and Mamajek's article in these proceeding for a recalibration including gyrochronology. It would therefore be appropriate to compare the new gyro ages with these older chromospheric ages. The best sample for this comparison is the Mt. Wilson sample of cool stars, one studied intensively for decades for chromospheric activity, and for which measured rotation periods are also available.

Fig. 6 shows this comparison, the cross indicating representative errors. The basic result to note is that there is reasonable agreement between the two ages because the upper left and lower right corners are unoccupied. A closer inspection shows that the chromospheric ages used here (Donahue 1998) are somewhat longer than the gyro ages, as the dashed median line shows. Dividing the stars into blue $(B-V<0.6)$, green $(0.6>B-V>0.8)$, and red $(B-V>0.8)$, shows that the discrepancy relates mostly to the blue F stars, whose lifetime of $5 \mathrm{Gyr}$ is indicated in the figure, as is the age of the universe.

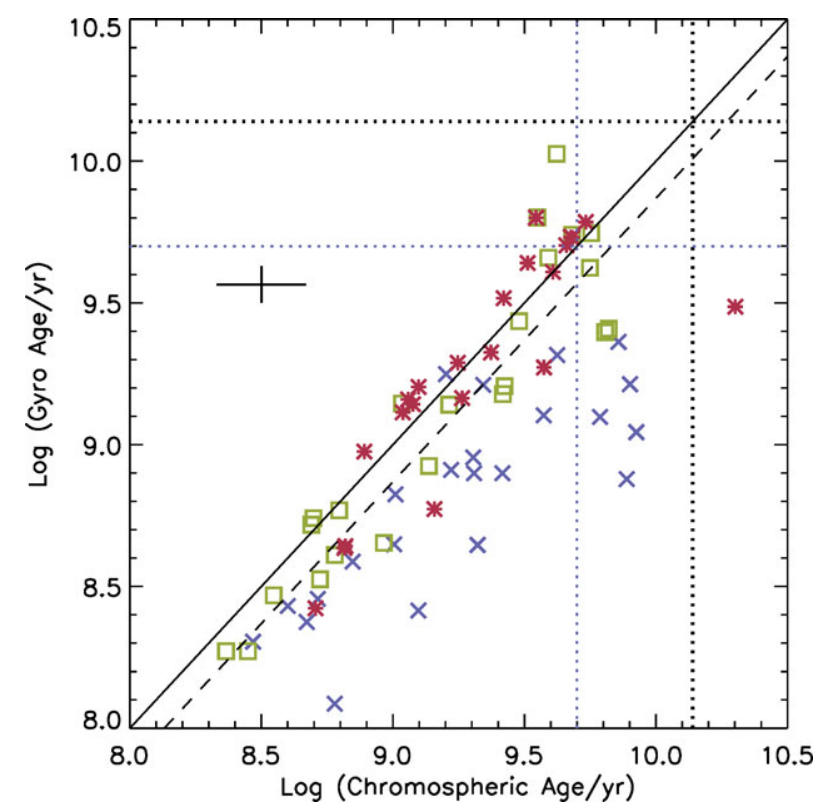

Figure 6. Comparison between gyro- and chromospheric ages for the Mt. Wilson star sample. Two conclusions can be drawn: (1) The two types of ages are in rough agreement, but the chromospheric ages are somewhat larger, and (2) The discrepancy relates mostly to the blue $\mathrm{F}$ stars (crosses) whose 5 Gyr lifetime is marked, rather than the green G stars (squares) or the red K stars (asterisks). (Figure from Barnes, 2007) 


\section{Comparison with isochrone ages}

An equivalent comparison of gyro- and isochrone ages demonstrates the difficulty of deriving isochrone ages for field stars.

The most modern and homogeneous field star isochrone ages available are those for the SPOCS star sample of Takeda et al. (2007), who have undertaken a Bayesian age analysis, based on the method of Pont \& Eyer (1994), and a prior uniform spectroscopic study of these stars by Valenti \& Fischer (2005). The stars in common with those in Barnes (2007) are displayed in Fig. 7.

Despite the Bayesian technique's admirable attempt to account for the asymmetric error distribution in color-magnitude diagrams, the isochrone ages are still on average a factor of $\sim 2.7$ larger than the gyro ages. Some upper and lower limits are included when they represent wide binary stars with measured rotation periods. Related components are connected with dashed lines. These only serve to underscore the difficulty of deriving isochrone ages for non-cluster main sequence stars.

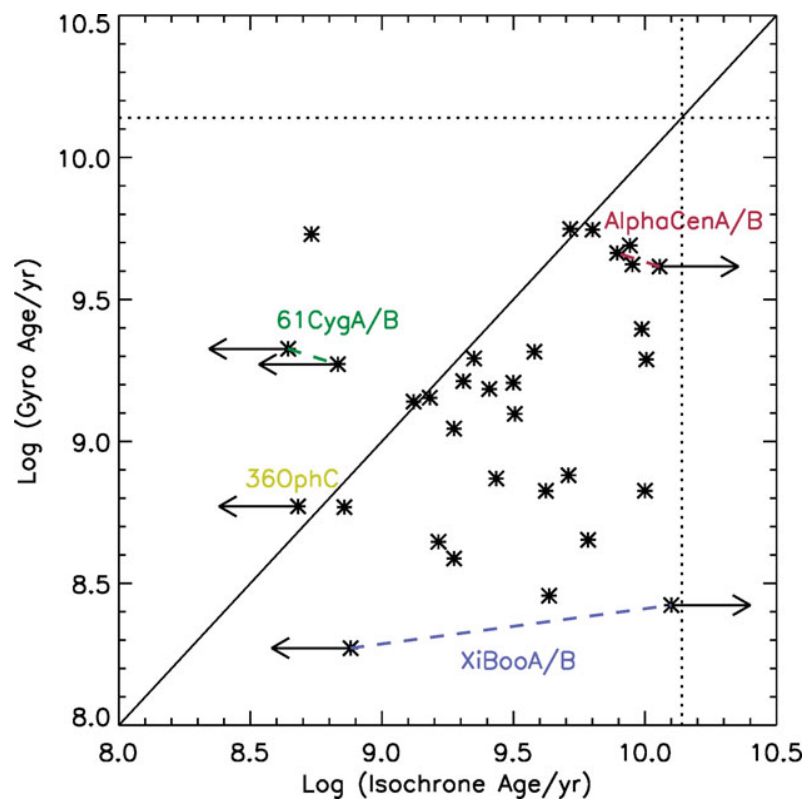

Figure 7. Comparison between gyro- and Bayesian isochrone ages for stars in common with the Takeda et al. (2007) SPOCS sample. The isochrone ages are on average $\sim 2.7$ times the gyro ages. Upper- and lower limits are included if they concern wide binaries with rotation periods. The components are connected by dashed lines. (Figure from Barnes, 2007)

\section{Ages for wide binaries}

Finally, we arrive at that very desirable property that an age determination method yield the same age for stars that we believe to be coeval. Indeed, there are a handful of wide binaries where rotation periods for both components have been measured. Thus, their ages may be determined independently. (We use wide binaries to be sure that there has been no tidal or magnetic hanky-panky between the components.)

The color-period diagram for the three available systems is shown in Fig. 8, along with the mean gyro isochrones and their errors for each pair, with details in Table 2 (from Barnes 2007). The ages for the components appear to be in agreement within the errors. 


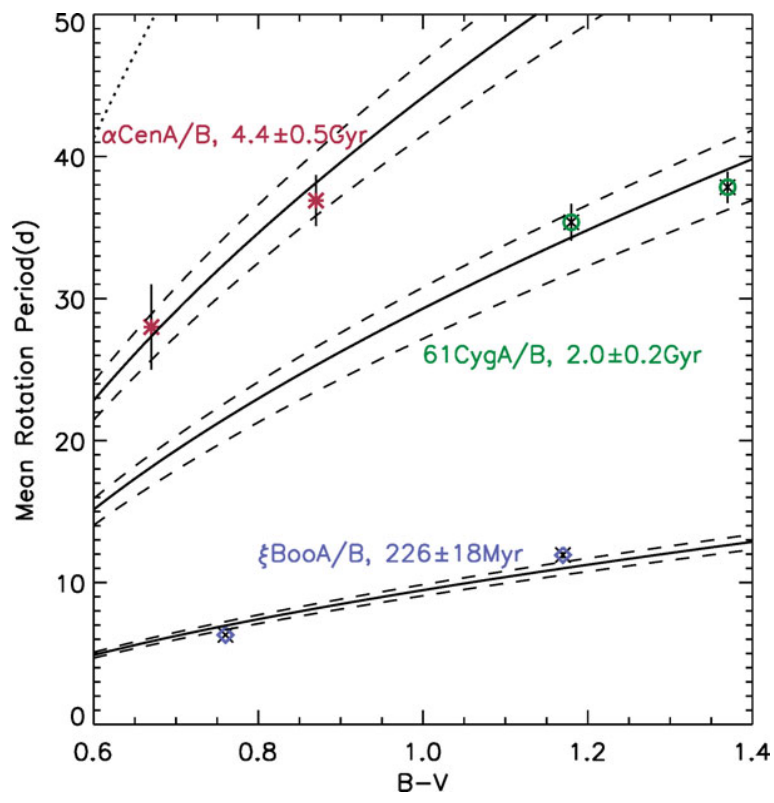

Figure 8. The components of the wide binaries $\xi$ Boo, $61 \mathrm{Cyg}$, and $\alpha$ Cen appear to give the same gyro ages. (Figure from Barnes, 2007)

The age of the 36 Oph triple system is taken to be that of the presumably non-interacting tertiary component.

\section{Conclusions}

In summary, we have constructed an improved method of determining the age of a main sequence star from its measured rotation period, calibrated it using the Sun, and shown that the associated errors are smaller than those from prior methods.

The key steps of the construction are:

- All late F-early M stars become I-type rotators within a couple of $100 \mathrm{Myr}$,

- Their rotation periods, $P$, are describable as a product of two separable functions, $f$, and $g$, of the $B-V$ color and age, $t$, respectively: $P(B-V, t)=f(B-V) \cdot g(t)$,

Table 2. Ages for wide binary systems

\begin{tabular}{|c|c|c|c|c|c|c|}
\hline System & Star & $B-V$ & $\bar{P}_{r o t}$ & Age chromo & Age $_{\text {iso }}$ & Age gyro \\
\hline \multirow{3}{*}{$\xi \mathrm{Boo}$} & HD131156A & 0.76 & $6.31(0.05)$ & $232 \mathrm{Myr}$ & $<760 \mathrm{Myr}$ & $187 \pm 21 \mathrm{Myr}$ \\
\hline & HD $131156 B$ & 1.17 & $11.94(0.22)$ & $508 \mathrm{Myr}$ & $>12600 \mathrm{Myr}$ & $265 \pm 28 \mathrm{Myr}$ \\
\hline & Mean & & & & & $226 \pm 18 \mathrm{Myr}$ \\
\hline \multirow{3}{*}{$61 \mathrm{Cyg}$} & HD 201091 & 1.18 & $35.37(1.3)$ & $2.36 \mathrm{Gyr}$ & $<0.44 \mathrm{Gyr}$ & $2.12 \pm 0.3 \mathrm{Gyr}$ \\
\hline & HD 201092 & 1.37 & $37.84(1.1)$ & $3.75 \mathrm{Gyr}$ & $<0.68 \mathrm{Gyr}$ & $1.87 \pm 0.3 \mathrm{Gyr}$ \\
\hline & Mean & & & & & $2.0 \pm 0.2 \mathrm{Gyr}$ \\
\hline \multirow{3}{*}{$\alpha$ Cen } & HD 128620 & 0.67 & $28(3)$ & $5.62 \mathrm{Gyr}$ & $7.84 \mathrm{Gyr}$ & $4.6 \pm 0.8 \mathrm{Gyr}$ \\
\hline & HD 128621 & 0.87 & $36.9(1.8)$ & $4.24 \mathrm{Gyr}$ & $>11.36 \mathrm{Gyr}$ & $4.1 \pm 0.7 \mathrm{Gyr}$ \\
\hline & Mean & & & & & $4.4 \pm 0.5 \mathrm{Gyr}$ \\
\hline \multirow{3}{*}{$36 \mathrm{Oph}$} & HD 155886 & 0.85 & $20.69(0.4)$ & $1.1 \mathrm{Gyr}$ & & $1.42 \pm 0.19 \mathrm{Gyr}$ \\
\hline & HD 155885 & 0.86 & $21.11(0.4)$ & $1.2 \mathrm{Gyr}$ & & $1.44 \pm 0.20 \mathrm{Gyr}$ \\
\hline & HD 156026 & 1.16 & $18.0(1.0)$ & $1.4 \mathrm{Gyr}$ & $<0.48 \mathrm{Gyr}$ & $0.59 \pm 0.07 \mathrm{Gyr}$ \\
\hline
\end{tabular}


- $f(B-V)$ and $g(t)$ can be determined empirically with small errors,

- $g(t)$ is such that initial variations become increasingly irrelevant with time,

- The functional dependence is easily inverted to get $t=t(P, B-V)$, and

- The age error, $\delta t=\delta t(t, P, B-V)$, is calculated.

The technique compares favorably with prior methods, which it complements, and passes some important tests. Precise time-series photometry is increasingly available from the ground and from space, making stellar rotation periods routinely measurable. Consequently, we recommend measuring rotation periods for appropriate main sequence cool stars where precise ages are desired, and using gyrochronology to derive them.

\section{References}

Baliunas, S., Sokoloff, D., \& Soon, W. et al. 1996, ApJL, 457, 99

Barnes, S. A. 1998, PhD Thesis, Yale University

Barnes, S. A. 2003, ApJ, 586, 464

Barnes, S. A. 2007, ApJ, 669, 1167

Demarque, P. D. \& Larson, R. B. 1964 ApJ, 140, 544

Donahue, R. A. 1998, in: R. A. Donahue \& Bookbinder, J. A. (eds.), Tenth Cambridge Workshop on Cool Stars, Stellar Systems and the Sun (San Francisco: ASP), p. 1235

Kawaler, S. D. 1989, ApJL, 343, 65

Mamajek, E. E. \& Hillenbrand, L. A. 2008, ApJ, 687, 1264

Meibom, S., Mathieu, R. D., \& Stassun, K. G. 2008, ApJ, in press (arXiv/astroph: 0805:1040)

Noyes, R. W., Hartmann, L. W., Baliunas, S. L., Duncan, D. K., \& Vaughan, A. H. 1984, ApJ, 279,763

Pizzolato, N., Maggio, A., Micela, G., Sciortino, S., \& Ventura, P. 2003, A\&\&A, 397, 147

Pont, F. \& Eyer, L. 1994, MNRAS, 351, 487

Radick, R. R., Thompson, D. T., Lockwood, G. W., Duncan, D. K. \& Baggett, W. E. 1987, ApJ, 321, 459

Sandage, A. 1962, ApJ, 135, 349

Skumanich, A. 1972, ApJ, 171, 565

Soderblom, D. R., Duncan, D. K., \& Johnson, D. R. H. 1991, ApJ, 375, 722

Strassmeier, K. G. Washuettl, A., Granzer, Th., Scheck, M., \& Weber, M. 2000, A $\& A S, 142$, 275

Takeda, G., Ford, E. B., Sills, A., Rasio, F. A., Fischer, D. A., \& Valenti, J.A. 2007, ApJS, 168, 297

Valenti, J. A. \& Fischer, D. A. 2005, ApJS, 159, 141

Wilson, O. C. $1963, A p J, 138,832$

\section{Discussion}

P. Goudfroois: Interesting method. However, it seems to me that for this method to work for any given star, one needs to know its foreground reddening very well, especially for the earlier- type stars where the "curvature" in the period-color relation is largest. I was wondering whether this issue could play a role in the apparent disagreement with ages from chromospheric lines.

S. BARNES: The disagreement with chromospheric ages is probably of a different origin. This is because reddening is not an issue for the (very close) Mount Wilson field stars. That said, there could be a residual effect in the calibration of gyrochronology if the cluster reddenings used are not quite correct.

E. Mamajek: How long does it take for a C-sequence star to "jump the gap" to the I-sequence? Is there a way to flag a star in the field as "gap" vs. "interface"? 
S. BARNES: It varies from zero (late-F stars in the youngest clusters are already on the I sequence) to a few $100 \mathrm{Myr}$ for early M stars. This includes a phase on the C-sequence. The transition from $\mathrm{C}$ to $\mathrm{I}$ is shorter because fewer stars are in the gap region in colorperiod diagrams, reminiscent of the Hertzsprung gap. Because of this timescale, any star above the $100 \mathrm{Myr}$ isochrone in the color-period diagram is basically guaranteed to be an I-sequence star. Eventually we may have other ways of distinguishing the two, perhaps from magnetic field considerations.

M. Pinsonneault: There is a theoretical context here: One expects a tight relationship between rotation and age to exist only after stars lose memory of their initial conditions. I also find it very worrying that the models disagree with the data where the isochrone ages can be tested. Your thoughts?

S. BARNES: It is correct that the relationship will be tight after stars lose memory of their initial conditions. This happens as $1 / t$. The data are what they are. The method is empirical. For the clusters, it does not really matter if their ages are slightly modified since they are all young. Any age error in there will simply show up as an increased spread in $f(B-V)$, which shows up as an increased error in the gyro age. The index $n$ depends on the solar calibration. As for the models, I do not think that they get the rotational evolution correct, or even that the physics included is completely correct. 


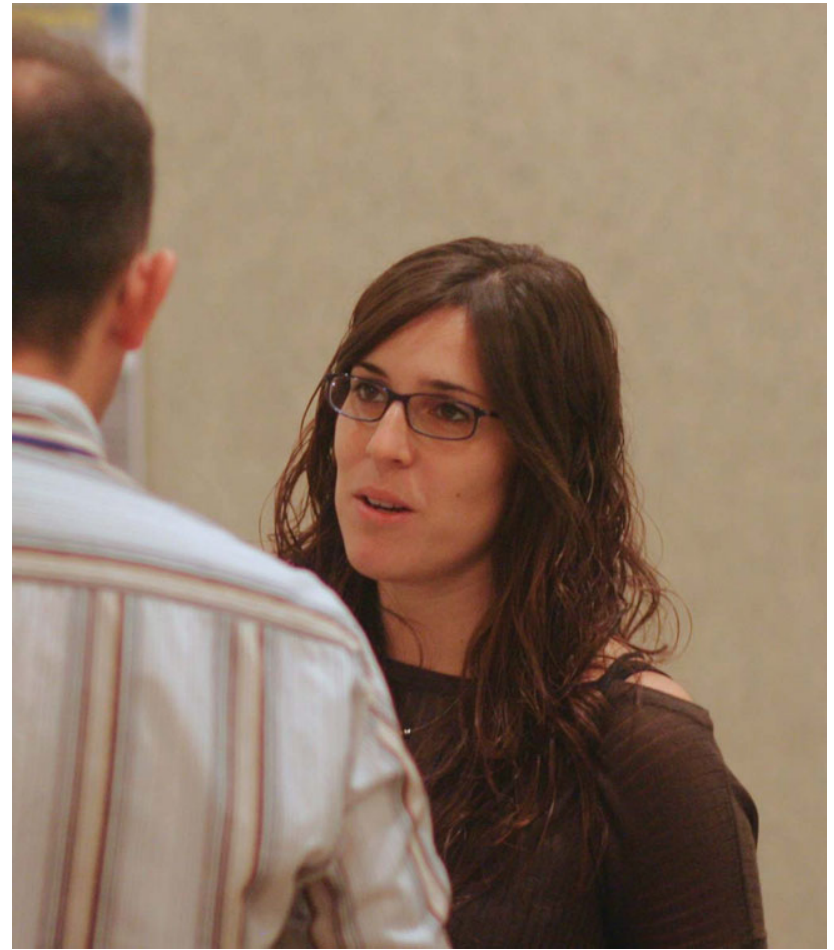

Theresa Antoja

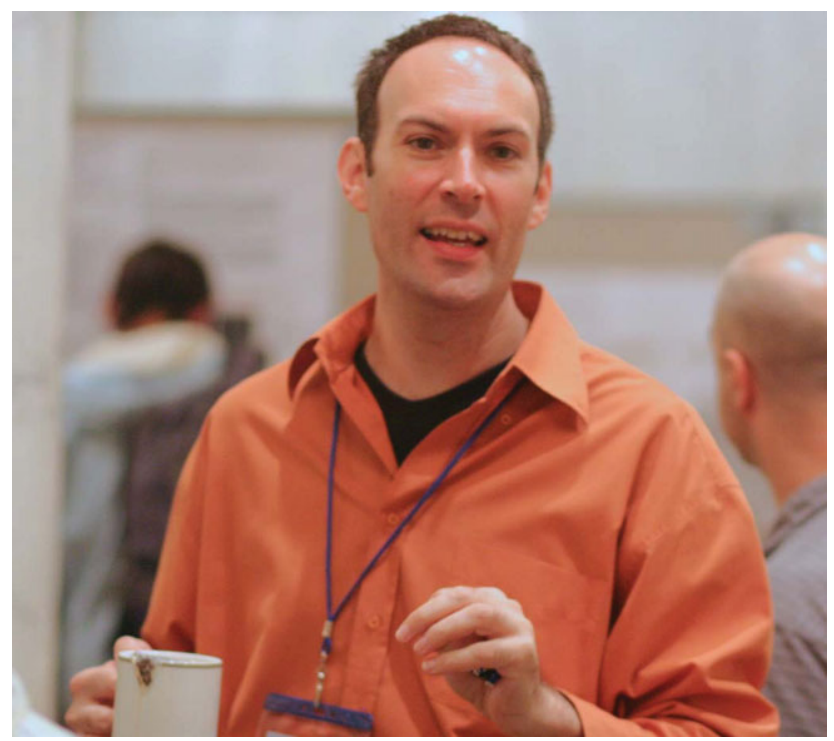

Guido De Marchi 